\title{
Injury of an aberrant vertebral artery during a routine corpectomy: a case report and literature review
}

\author{
MS Eskander, PJ Connolly, JP Eskander and DD Brooks \\ Department of Orthopedics, UMass Medical School, Worcester, MA, USA
}

\begin{abstract}
Study design: A case report of a 58-year-old man who sustained a laceration of his left vertebral artery during a routine corpectomy for cervical myelopathy is reported.

Objective: To report iatrogenic injury of a tortuous vertebral artery during anterior cervical spine surgery and discuss appropriate diagnosis and treatment options for this complication.

Setting: UMass Memorial Medical Center, Worcester, MA, USA.

Background data: Vertebral artery anomalies, although rare, are typically present with degenerative processes and great care must be taken to avoid damage during a corpectomy. Cross-sectional imaging coupled with intraoperative angiography is helpful for the urgent evaluation of the injury site and identification of the contralateral vertebral artery's status.

Methods: This is a single case of a patient sustaining a laceration of the left vertebral artery during surgery, which resulted in a lateral medullary stroke.

Results: After the left vertebral artery laceration, hemostasis was achieved. With the intent to better visualize and possibly embolize or stent the injury, an angiographic study was carried out. The angiogram revealed a laceration of the left vertebral artery within the vertebral foramina at vertebral body level C6, but intact distal flow. The patient underwent angiographic embolization and a subsequent magnetic resonance imaging (MRI) revealed a left lateral medullary stroke consistent with the lack of flow through the left vertebral artery from $\mathrm{C} 6$ to the basilar artery.

Conclusion: If a tortuous vertebral artery is suspected, then meticulous review of preoperative crosssectional imaging should be implemented along with angiographic examination. If anomalies are detected and the standard procedure cannot be safely carried out, then alterations, such as preoperative stent placement, need to be considered.

Spinal Cord (2009) 47, 773-775; doi:10.1038/sc.2009.29; published online 24 March 2009
\end{abstract}

Keywords: aberrant vertebral artery; corpectomy; cervical myelopathy; angiography; medullary stroke

\section{Case report}

This is a case report of a 58-year-old man with an iatrogenic laceration of a tortuous left vertebral artery. This complication occurred during a routine corpectomy for cervical myelopathy.

Upon initial presentation, the patient noted progressive difficulty maintaining the ambulatory balance and performing fine motor activities. The examination was significant for a broad shuffling gait. Babinski testing revealed dorsiflexion on the right and an equivocal response on the left. He had brisk and symmetric patellar reflexes, absent Achilles reflexes and no clonus. He had normal upper extremity reflexes and motor examination. Motor examination of his lower

Correspondence: Dr MS Eskander, Department of Orthopedics, UMass Medical School, 55 Lake Ave North, Worcester, MA 1605, USA,

E-mail: eskanm01@ummhc.org

Received 15 December 2008; revised 19 February 2009; accepted 24 February 2009; published online 24 March 2009 extremities revealed 4/5 proximal lower extremity weakness, but full distal strength.

A cervical and thoracic magnetic resonance imaging (MRI) revealed C6-7 posterior disc herniation with myelomalacia. Superior migration of the central disc herniation to the midregion of the C6 body was noted. For treatment, a standard Southwick and Robinson approach ${ }^{1}$ to the cervical spine was implemented for C6 corpectomy and C5-7 fusion. Once the C5-6 and C6-7 discectomies were completed, the uncovertebral joints were used to determine the width of the corpectomy and a high-speed drill was used for completion of the procedure. As the drill was advanced laterally, an inordinate amount of bleeding occurred. A vascular surgeon was involved in achieving hemostasis. An angiography was carried out to better visualize and treat the vertebral artery injury. The angiogram (Figure 1) revealed laceration of the left vertebral artery within the vertebral foramina at vertebral body level C6, but intact distal flow. The artery followed an 


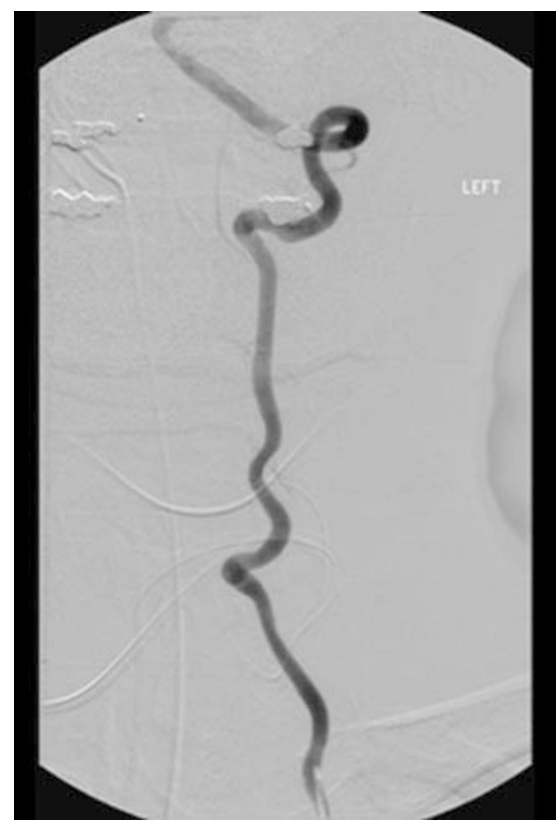

Figure 1 Angiogram of the tortuous left vertebral artery.

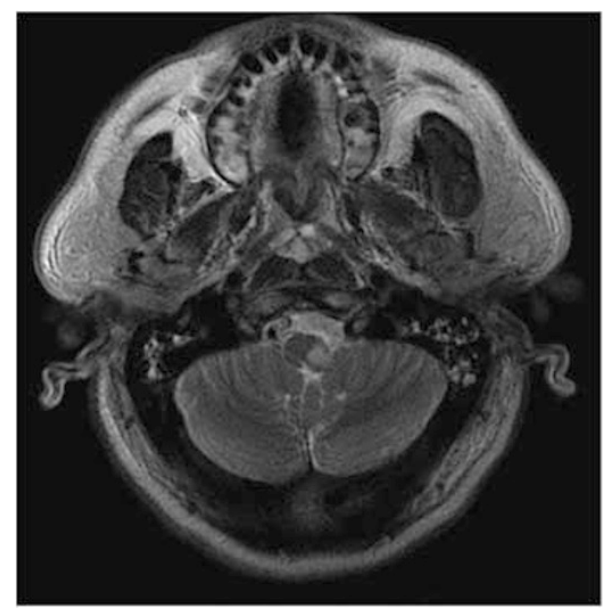

Figure 2 Axial T2 MRI showing left lateral medullary signal intensity. MRI, magnetic resonance imaging.

aberrant course within the vertebral foramina at C6. The patient then underwent complete embolization resulting in occlusion of the left vertebral artery below the posterior inferior cerebellar artery origin and also at C6. After the embolization procedure, the corpectomy was completed and the following instrumentation was uneventful. Bony dissection was kept to a minimum on the right side of the C6 body to avoid further injury.

Postoperatively, left eyelid drooping was noted. A subsequent MRI (Figure 2) of the patient's brain revealed a lateral medullary stroke consistent with Wallenberg's syndrome (lateral medullary syndrome).

Imaging indicates that the vertbral artery anomaly is at the C6 midbody level. The axial MRI and CT (Figures 3 and 4) reveals midline migration of the left vertebral artery. In

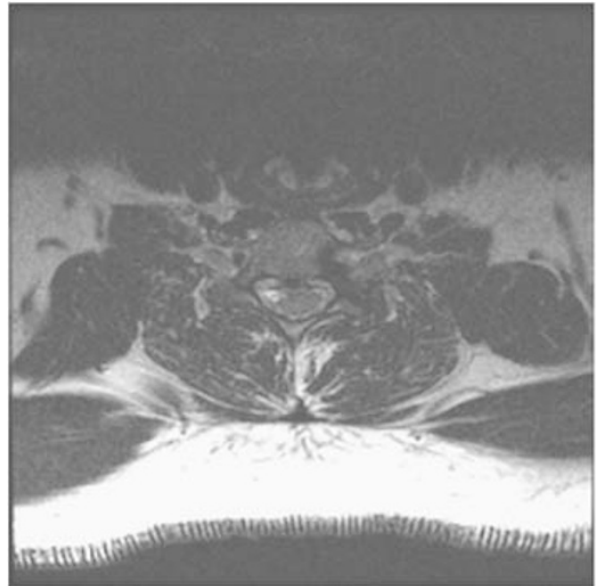

Figure 3 Preoperative axial T2 MRI showing the left vertebral artery migrating into the $\mathrm{C} 6$ body. MRI, magnetic resonance imaging.

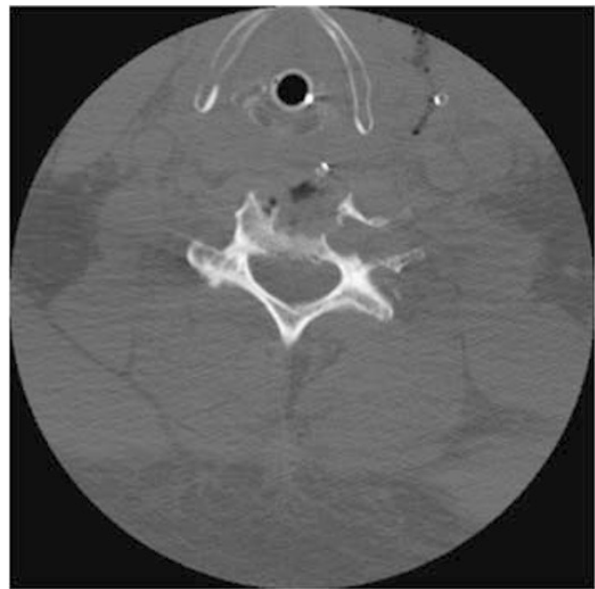

Figure 4 Postcorpectomy axial CT showing the communication between the corpectomy and the foramen. CT, computed tomography.

retrospect, after meticulous review of these images, one could have predicted an aberrant vertebral artery pathway and then referred to angiography for better assessment of the artery's tortuous route.

\section{Discussion}

There are several theories behind abnormal vertebral artery tortuosity and midline migration. One proposed etiology follows the logic that as degenerative osteoarthritic changes of the cervical spine occur over time, the vertebral artery adapts accordingly by developing a tortuous route and eroding posterolateral vertebral bodies. ${ }^{2}$ This portion of the vertebrae is more susceptible to erosion than the endplate due to a lower bone density. Additionally, pathologic conditions, such as tumors, infections, non-unions and systemic diseases can serve as predisposing factors for the development of bone erosion. ${ }^{2}$ 
Interestingly, available data suggest that anomalous vertebral arteries occur more often on the patient's left side. The literature reports eight on the left and two on the right at the levels of C3 (three cases), C4 (five cases) and C6 (two cases). ${ }^{2-4}$

When preoperative axial imaging reveals a degenerative or pathologic picture, heightened awareness of an aberrant vertebral artery is warranted. It is imperative that the surgeon reviews computed tomographies (CTs) and MRIs preoperatively to determine whether an abnormally medial vertebral artery is present. When an anomaly threatens the safety of the standard procedures, then the surgical plan may need to be altered or modified. Appropriate modifications may include a less aggressive corpectomy method or a posterior-based procedure. Another consideration would be to preoperatively stent the artery to protect the vessel from inadvertent injury.

Using well-defined surgical landmarks, unplanned contact with the vertebral artery is successfully avoided in routine cases. The vertebral artery is most vulnerable to injury anteriorly to C7, laterally at C3-C7 and posteriorly at C1 and C2. Reasons for accidental injury include aggressive lateral burring, weakened bone and anomalous vertebral artery midline migration.

In the presence of a tortuous vertebral artery, the standard landmarks may not prevent iatrogenic injury. Failure to recognize this anomaly during preoperative planning can lead to laceration of the vessel. Therefore, a case-by-case review of imaging is necessary to determine the patient's specific anatomy.

The treatments for laceration of the vertebral artery consist of direct pressure tamponade, direct electrocoagulation, direct exposure with vascular clips, transosseous ligation and direct exposure with ligation or repair. ${ }^{5}$ A recent report described endovascular techniques and offer a more refined algorithm for dealing with this type of injury. ${ }^{2}$ Intraoperative angiography will help the urgent evaluation of the injury site and identify the contralateral vertebral artery status. If a patent contralateral vertebral artery or a sufficient collateral posterior circulation is present, direct occlusion of an injured vertebral artery is justified. However, if the patient has a hypoplastic contralateral vertebral artery, then vessel repair or stenting is preferred. ${ }^{2,6}$ Unfortunately, quick action and appropriate measures may not completely preclude side effects as our patient developed a lateral medullary stroke after his vertebral artery injury and subsequent embolization.

If the anomaly is recognized before surgery, two promising alternative approaches have been identified. Depending on the pathology present, treatment options include combining a cervical discectomy at the anomalous level with a corpectomy at other levels for focal disease. Or, in case of multilevel anomalies, a posterior approach appears to be the safest treatment course. . $^{3,4}$

\section{Conclusions}

On account of the numerous complications that can result from a vertebral artery injury, it is imperative to control bleeding. Cross-sectional MRI and bone target CT imaging should effectively locate the position and path of the vertebral artery in question. Ultimately, timely diagnosis and treatment of the potential complications can be expedited with close monitoring in an intensive care unit. Also, when appropriate, preventive measures, such as preoperative stent placement, deserve consideration.

\section{References}

1 Southwick WO, Robinson RA. Surgical approaches to the vertebral bodies in the cervical and lumbar regions. J Bone Join Surg (Am) 1976; 39: 631-644.

2 Choi JW, Lee JK, Moon KS et al. Endovascular embolization of iatrogenic vertebral artery injury during anterior cervical spine surgery report of two cases and review of the literature. Spine 2006; 31: E891-E894.

3 Curylo LJ, Mason HC, Bohlman HH et al. Tortuous course of the vertebral artery and anterior cervical decompression: a cadaveric and clinical case study. Spine 2000; 25: 2860-2864.

4 Oga M, Yuge I, Terada K et al. Tortuosity of the vertebral artery in patients with cervical spondylotic myelopathy. Spine 1996; 21: $1085-1089$.

5 Smith MD, Emery SE, Dudley A et al. Vertebral artery injury during anterior decompression of the cervical spine. A retrospective review of ten patients. J Bone Joint Surg (Br) 1993; 75: 410-415.

6 Garcia AM, Rosahl SK, Lehmberg J et al. Life-threatening bleeding from a vertebral artery pseudoaneurysm after anterior cervical spine approach: endovascular repair by a triple stent-in-stent method. Case report. Neuroradiology 2005; 47: 282-286. 